\begin{tabular}{|c|l|}
\hline Title & Coulomb staircase and total spin in quantum dots \\
\hline Author(s) & A kera, Hiroshi \\
\hline Citation & $\begin{array}{l}\text { Physical Review B, 60/15), 10683_10686 } \\
\text { https://doi.org/10.1103/PhysRevB.60.10683 }\end{array}$ \\
\hline Issue Date & 1999-10-15 \\
\hline Doc URL & http://hdl.handle.net/2115/6058 \\
\hline Rights & Copyright $\odot 1999$ A merican Physical Society \\
\hline Type & article \\
\hline File Information & PRB60_15.pdf \\
\hline
\end{tabular}

Instructions for use 


\title{
Coulomb staircase and total spin in quantum dots
}

\author{
Hiroshi Akera \\ Department of Applied Physics, Hokkaido University, Sapporo 060-8628, Japan
}

(Received 19 May 1999)

\begin{abstract}
The dependence of the Coulomb staircase on the total spin of a quantum dot is calculated at a current step corresponding to a transition between the ground states of two successive electron numbers. The ratio of the step heights for the positive and negative bias is simply the ratio of the spin degeneracies of the ground states, when tunneling rates across the two barriers are strongly asymmetric. This can be used for determining experimentally the total spin and therefore identifying the spin blockade. The in-plane magnetic-field dependence of the Coulomb staircase is also calculated, and it is shown that the relative height of spin-split steps can be used alternatively to determine the total spin. [S0163-1829(99)11639-4]
\end{abstract}

Growing interest has been attracted to transport through a quantum $\operatorname{dot}^{1,2}$ and has recently been extended to roles of the spin degree of freedom in this system. A transition of a spin singlet to triplet state, ${ }^{3,4}$ a triplet state due to Hund's rule, ${ }^{5}$ and the even larger spin polarization ${ }^{6,7}$ have been observed. The Kondo effect has been observed when the localized spin in the dot interacts with conduction-electron spins in leads. ${ }^{8,9}$ The decoherence of the single-electron phase due to spin flips at a dot has been studied theoretically. ${ }^{10}$ Another prominent example is the spin blockade ${ }^{11,12}$ studied theoretically by many authors. ${ }^{11-16}$ The spin blockade is the disappearance of a peak in the Coulomb oscillations of the linear conductance, which occurs when the total spins $S$ and $\widetilde{S}$ of the ground states for $N$ and $N+1$ electrons in the dot do not satisfy the spin selection rule $\widetilde{S}=S \pm 1 / 2$, which can occur due to many-body effects. In spite of considerable experimental efforts made, there has not yet been success in obtaining decisive evidence of the spin blockade. Although it is necessary to know the total spins $S$ and $\widetilde{S}$ to identify the spin blockade, most experimental assignments of the spin have been made indirectly from the addition spectrum reflecting the orbital character ${ }^{3-7}$ and the $\operatorname{spin}^{17}$ of each added electron and leave a limitation in determining the total spin. This paper shows that the relative step heights of the Coulomb staircase can determine directly the total spin of the ground state for any electron numbers.

The step height of the Coulomb staircase, or the saturation current, is in general different between the positive and the negative bias and reflects the degeneracy of the states relevant to the transition. This has been shown theoretically first for the case with a single orbit ${ }^{18,19}$ and extended ${ }^{13}$ to the case with $M$ orbits $(M>1)$. The saturation current at the transition between $N$ and $N+1$ for the positive and negative bias is $I_{+}=(-e / \hbar)(M-N) \gamma_{e}(N+1) \gamma_{c} /\left[(M-N) \gamma_{e}+(N\right.$ $\left.+1) \gamma_{c}\right]$ and $I_{-}=(-e / \hbar) N \gamma_{e}(M-N+1) \gamma_{c} /\left[N \gamma_{e}+(M\right.$ $\left.-N+1) \gamma_{c}\right]$ with $\gamma_{e}$ and $\gamma_{c}$ the level broadening due to tunneling into the emitter and the collector, respectively. This is the case even in the presence of many-body effects in states in the dot. The result becomes particularly simple when $\gamma_{e}$ $\ll \gamma_{c}$ to give $I_{+} / I_{-}=(M-N) / N$.

In this paper we consider the Coulomb-staircase step at the transition between the $N$-electron ground state with spin
$S$ and the $N+1$-electron ground state with spin $\widetilde{S}$ and show that $I_{+} / I_{-}$is simply the ratio of their spin degeneracies $(2 \widetilde{S}+1) /(2 S+1)$. We employ the following assumptions in our calculation. (i) In the nonlinear regime with different chemical potentials in the emitter and the collector, the electron distribution in the dot depends on $\gamma_{e}$ and $\gamma_{c}$, and the current is, in general, a complicated function of $\gamma_{e}$ and $\gamma_{c}$. Therefore, we assume asymmetric tunneling rates such that $\gamma_{e} \ll \gamma_{c}$. Owing to this, the electron distribution in the dot is determined by the collector chemical potential. (ii) We also employ, for simplicity, an assumption that the tunneling is weak so that the level broadening is much smaller than the thermal energy. However, it may not be essential to the final result, if correlations such as in the Kondo effect are not important.

We also calculate the magnetic-field dependence of the current for the magnetic field parallel to the two-dimensional plane. We assume that (iii) the in-plane magnetic field affects only the spin degree of freedom in our two-dimensional electron systems. It is shown that the normalized current at a step of the Coulomb staircase is a universal function ${ }^{21}$ that depends only on $S, \widetilde{S}$, the spin splitting, and the chemical potentials of two leads divided by the thermal energy. A variety of methods to determine the total spin are available from this universal function: for example, employing the relative step heights of the spin-split Coulomb staircase.

Our Hamiltonian $H$ consists of $H_{d}$ for the dot, $H_{L}$ for the leads, and $H_{t}$ for tunnelings:

$$
\begin{gathered}
H=H_{d}+H_{L}+H_{t}, \\
H_{d}=\sum_{n, \sigma} \varepsilon_{n \sigma} c_{n \sigma}^{\dagger} c_{n \sigma}+H_{\mathrm{int}}, \\
H_{L}=\sum_{l, k, \sigma} \varepsilon_{l k \sigma} c_{l k \sigma}^{\dagger} c_{l k \sigma}, \\
H_{t}=\sum_{l, k, n, \sigma}\left(V_{l k n} c_{l k \sigma}^{\dagger} c_{n \sigma}+\text { H.c. }\right),
\end{gathered}
$$

where $c_{n \sigma}^{\dagger}\left(c_{n \sigma}\right)$ are creation (annihilation) operators for an electron with spin $\sigma$ in orbit $n$ in the dot, and $c_{l k \sigma}^{\dagger}\left(c_{l k \sigma}\right)$ are 
those for an electron in state $k$ in lead $l(l=e$ for the emitter and $l=c$ for the collector). The spin splitting produced by the in-plane magnetic field is reflected in the $\sigma$ dependence of energy levels $\varepsilon_{n \sigma}$ and $\varepsilon_{l k \sigma} . H_{\text {int }}$ is the interaction term whose explicit form is not necessary in the following general discussion. We only need to assume that the energy separation between the ground-state multiplet and the first excited state for $N$ electrons in the dot and that for $N+1$ electrons are larger than the thermal energy, so that the current is exclusively due to transitions between the ground states for the two electron numbers. Each state in the ground-state multiplet (spin $S$ ) is labeled by the $z$ component of the spin $S_{z}$.

In the weak-tunneling regime we consider, the level broadening due to tunneling is much smaller than the thermal energy, so that electronic states and electron distribution in leads are little modified by tunnelings and correlations between the leads and the dot, such as in the Kondo effect, can be neglected. In this regime we can calculate the current by applying our previous theory, ${ }^{13}$ which employs the transition-rate formula for electron tunnelings between the two leads by incorporating the finite level broadening of intermediate many-body states. This method gives the same result as obtained from the master equation, or the kinetic equation. $^{18,23,24}$

In calculating the transition rate, the initial state is $|i\rangle$ $=\left|e k \sigma, L ; N, S S_{z}\right\rangle$ in which $e k \sigma$ state is occupied and $|L\rangle$ representing the occupation of the other levels in the two leads. One of intermediate states is $|m\rangle=\left|L ; N+1, \widetilde{S} \widetilde{S}_{z}\right\rangle$ with $\widetilde{S}_{z}=S_{z}+\sigma / 2$. The final state is $|f\rangle=\left|c k^{\prime} \sigma^{\prime}, L ; N, S S_{z}^{\prime}\right\rangle$ with $S_{z}^{\prime}+\sigma^{\prime} / 2=S_{z}+\sigma / 2$. The transition rate is given by

$$
P_{i, f}=\frac{2 \pi}{\hbar}|\langle i|\hat{T}| f\rangle|^{2} \delta\left(E_{i}-E_{f}\right)
$$

with

$$
\langle i|\hat{T}| f\rangle=\left\langle i\left|H_{t}\right| m\right\rangle\langle m|G| m\rangle\left\langle m\left|H_{t}\right| f\right\rangle .
$$

The propagator is defined by $G=\left(E_{i}-H+i \eta\right)^{-1}$ with $\eta$ the positive infinitesimal. $\langle m|G| m\rangle$ is replaced by its average with respect to $|L\rangle$ at the local equilibrium of the leads and evaluated in the weak-tunneling regime using the noncrossing approximation. ${ }^{25,26}$

The current is then expressed as

$$
\begin{aligned}
I= & -e \sum_{k, \sigma, S_{z}} \sum_{k^{\prime}, \sigma^{\prime}, S_{z}^{\prime}}\left[P_{i, f} f_{e k \sigma}\left(1-f_{c k^{\prime} \sigma^{\prime}}\right) g_{N S_{z}}\right. \\
& \left.-P_{f, i} f_{c k^{\prime} \sigma^{\prime}}\left(1-f_{e k \sigma}\right) g_{N S_{z}^{\prime}}\right],
\end{aligned}
$$

where $f_{l k \sigma}=f_{l}\left(\varepsilon_{l k \sigma}\right)=\left\{1+\exp \left[\left(\varepsilon_{l k \sigma}-\mu_{l}\right) / k_{B} T\right]\right\}^{-1}$ is the Fermi distribution function with the chemical potential $\mu_{l}$, and $g_{N S_{z}}$ is the probability of finding the $N$-electron state with $z$ component of spin $S_{z}$. Since the calculation of the current is made up to the first order of $\gamma_{e}, g_{N S_{z}}$ is evaluated in the zeroth order of $\gamma_{e}$, that is, at equilibrium with the collector and is given by $g_{N S_{z}}=\exp \left[-\left(E_{N S_{z}}-N \mu_{c}\right) / k_{B} T\right] / Z_{d}$ with $Z_{d}$ given by $\Sigma_{S_{z}} g_{N, S_{z}}+\Sigma_{S_{z}} g_{N+1, \tilde{S}_{z}}=1$. Similarly $\langle m|G| m\rangle$ in Eq. (3) is evaluated in the zeroth order of $\gamma_{e}$.
In the absence of spin-orbit interactions, dependences on $\sigma, S_{z}$, and $\widetilde{S}_{z}$ of matrix elements of $H_{t}$ are expressed using the Clebsch-Gordan coefficient since

$$
\left\langle N, S S_{z}\left|c_{n \sigma}\right| N+1, \widetilde{S} \widetilde{S}_{z}\right\rangle=\left\langle S S_{z} \frac{1}{2} \frac{\sigma}{2} \mid \widetilde{S} \widetilde{S}_{z}\right\rangle h_{n N S \tilde{S}},
$$

with $h_{n N S \tilde{S}}$ independent of $\sigma, S_{z}$, and $\widetilde{S}_{z}$. We neglect $k$ dependence of $V_{l k n}$ and the energy dependence of the density of states (per spin) $\rho_{l}$ in lead $l$ in its narrow range of interest (of the order of the spin splitting and the thermal energy) and introduce a constant describing the level broadening of the many-body states:

$$
\Gamma_{l N S \tilde{S}}=2 \pi \rho_{l}\left|\sum_{n} V_{l n} h_{n N S \tilde{S}}\right|^{2} .
$$

Finally, the current around the corresponding step is expressed as

$$
I=C_{I} F_{I}\left(S, \widetilde{S}, y_{e}, y_{c}, b\right)
$$

$\left[y_{l}=\left(\mu_{l}-\varepsilon_{0}\right) / k_{B} T\right.$ and $\left.b=g * \mu_{B} B / k_{B} T\right]$ with a universal function

$$
\begin{aligned}
F_{I}= & \sum_{\sigma S_{z}}\left|\left\langle S S_{z} \frac{1}{2} \frac{\sigma}{2} \mid \widetilde{S} \widetilde{S}_{z}\right\rangle\right|^{2}\left(g_{N, S_{z}}+g_{N+1, \widetilde{S}_{z}}\right) \\
& \times\left[f_{e}\left(\varepsilon_{\sigma}\right)-f_{c}\left(\varepsilon_{\sigma}\right)\right]
\end{aligned}
$$

$\left(\tilde{S}_{z}=S_{z}+\sigma / 2\right)$ and a prefactor

$$
C_{I}=\frac{(-e)}{\hbar} \Gamma_{e N S \tilde{S}}
$$

The addition energy $\varepsilon_{\sigma}$ is given by $\varepsilon_{\sigma}=E_{N+1, \tilde{S}_{z}}-E_{N, S_{z}}$ $=\varepsilon_{0}+g * \mu_{B} B \sigma / 2$, and $\varepsilon_{0}$ is that in the absence of the magnetic field. The prefactor $C_{I}$ depends on tunneling matrix elements and the ground-state wave functions with $N$ and $N+1$ electrons. Because of the rule for the addition of two spins, we have $\widetilde{S}=S \pm 1 / 2$. Results for $\widetilde{S}=S+1 / 2$ and for $\widetilde{S}$ $=S-1 / 2$ are related by

$$
\begin{aligned}
(2 S & +1) F_{I}\left(S, \widetilde{S}, y_{e}, y_{c}, b\right) \\
& =-(2 \widetilde{S}+1) F_{I}\left(\widetilde{S}, S,-y_{e},-y_{c}, b\right) .
\end{aligned}
$$

The above formula for the current is also applicable to materials with the valley degree of freedom $v$, in which singleelectron states in leads are labeled by $k$ and $\mathrm{v}$, if we redefine the constant as

$$
\Gamma_{l N S \tilde{S}}=\sum_{\mathrm{v}} 2 \pi \rho_{l \mathrm{v}}\left|\sum_{n} V_{l \mathrm{v} n} h_{n N S \tilde{S}}\right|^{2},
$$

where $n$ labels all single-particle states in the dot.

The step height of the normalized current $F_{I}$ has simple analytical expressions. First consider the following two cases. (i) Positive bias. The current is saturated when the emitter chemical potential $\mu_{e}$ is well above the addition energies $\varepsilon_{\sigma}$ of the $N+1$ th electron and the collector chemical potential $\mu_{c}$ is well below them. In this case the electron 

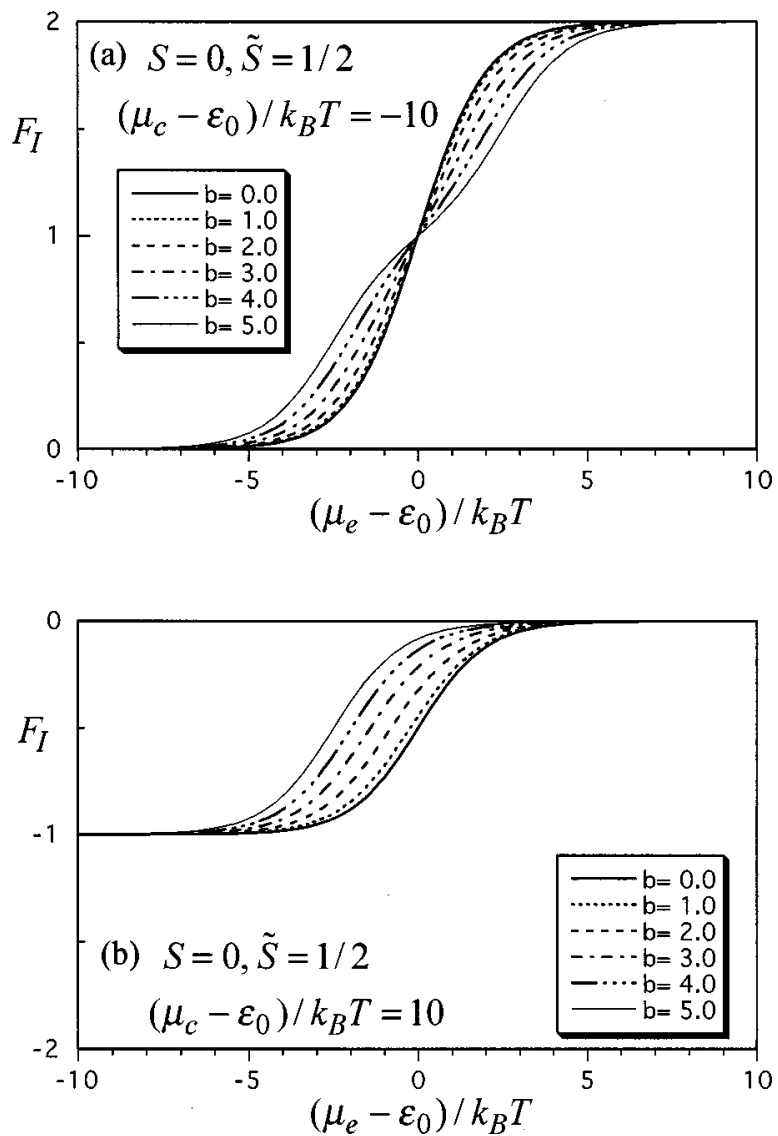

FIG. 1. Normalized current $F_{I}$ through a quantum dot for $S$ $=0$ and $\widetilde{S}=1 / 2$ as a function of the emitter chemical potential $\mu_{e}$ and the spin splitting $b=g^{*} \mu_{B} B / k_{B} T$ ( $S$ and $\widetilde{S}$ are the spins in $N$ and $N+1$-electron ground states, respectively). (a) Positive bias. The collector chemical potential $\mu_{c}$ is at $\varepsilon_{0}-10 k_{B} T$. (b) Negative bias. $\mu_{c}=\varepsilon_{0}+10 k_{B} T$.

number in the dot is close to $N$ since the electron distribution is determined by the collector chemical potential due to the assumed asymmetric tunneling rates $\left(\gamma_{e} \ll \gamma_{c}\right)$. Although the electron distribution among different $S_{z}\left(g_{N S_{z}}\right)$ depends on $b$, the saturation current is independent of $b$ and is obtained as

$$
F_{I}\left(S, \widetilde{S}, y_{e}, y_{c}, b\right)=\frac{2 \widetilde{S}+1}{2 S+1} .
$$

(ii) Negative bias. The saturation current when $\mu_{e}$ is well below $\varepsilon_{\sigma}$ and $\mu_{c}$ is well above them is similarly obtained as

$$
F_{I}\left(S, \widetilde{S}, y_{e}, y_{c}, b\right)=-1,
$$

independent of $b$. Experimently, we measure the ratio of the saturation current between the positive and the negative bias $I_{+} / I_{-}$, which is to be equal to $(2 \widetilde{S}+1) /(2 S+1) . \widetilde{S}=S$ $+1 / 2$ for $I_{+} / I_{-}>1$, while $\widetilde{S}=S-1 / 2$ for $I_{+} / I_{-}<1$. In both cases $S$ and $\widetilde{S}$ are determined.

An additional current step appears as a function of $\mu_{e}$ when the spin splitting is large enough $\left(b \gg 1\right.$ and $\left.S_{z}=S\right)$, depending on $\mu_{c}$ and $\widetilde{S}=S \pm 1 / 2$. When $\mu_{c}$ is well below $\varepsilon_{\sigma}$, the Coulomb staircase splits into two steps for $\widetilde{S}=S$
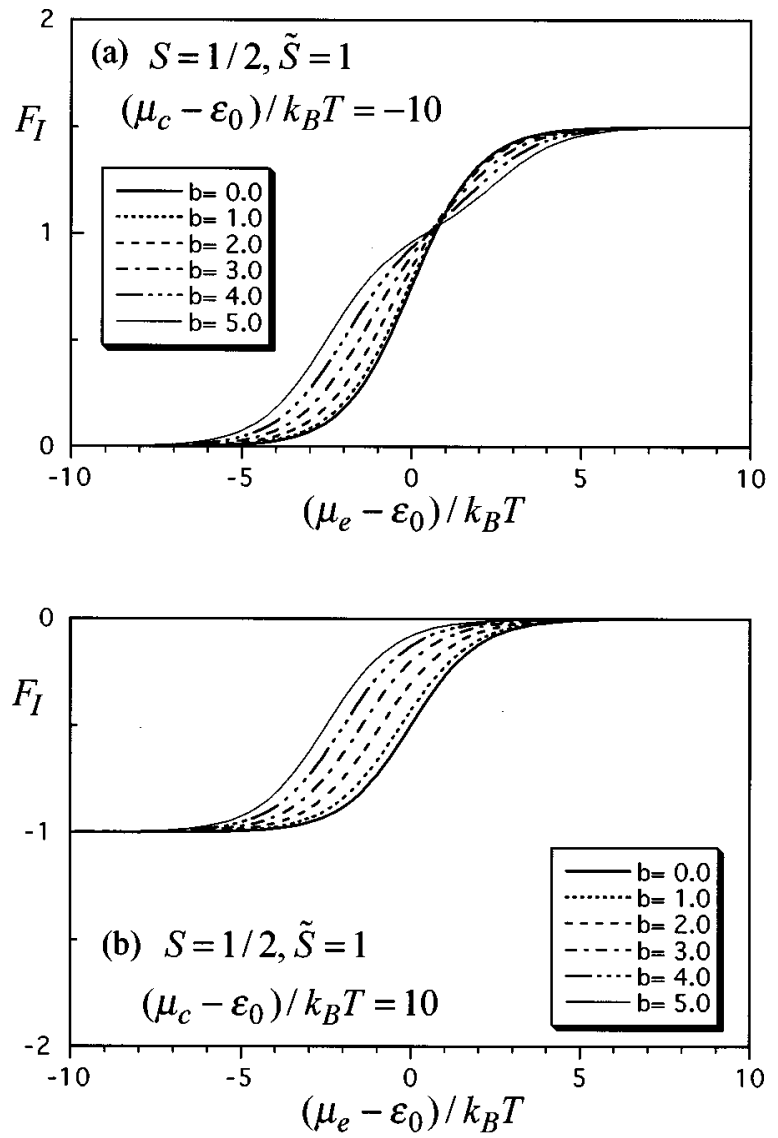

FIG. 2. Normalized current $F_{I}$ through a quantum dot for $S$ $=1 / 2$ and $\widetilde{S}=1$.

$+1 / 2$, while it does not split for $\widetilde{S}=S-1 / 2$. This has been used $^{27,20}$ to distinguish between $\widetilde{S}=S+1 / 2$ and $\widetilde{S}=S-1 / 2$. The step height at $\varepsilon_{\uparrow}<\mu_{e}<\varepsilon_{\downarrow}$ is shown as

$$
\begin{aligned}
F_{I}\left(S, \widetilde{S}, y_{e}, y_{c}, b\right) & =1, \text { for } \tilde{S}=S+1 / 2, \\
& =0, \text { for } \tilde{S}=S-1 / 2 .
\end{aligned}
$$

The vanishing step height occurs in the latter case because of the violation of the spin selection rule for the lower addition energy with $\sigma=+1 \quad\left(S_{z}+\sigma / 2=S+1 / 2>\widetilde{S}\right.$ with $\left.S_{z}=S\right)$. For $\widetilde{S}=S+1 / 2$, the step of the Coulomb staircase as a function of $\mu_{e}$ splits into $1:(2 S+1)^{-1}$, which can also be used to determine the total spin. ${ }^{28}$ When $\mu_{c}$ is well above $\varepsilon_{\sigma}$, on the other hand, the step splits into $1:(2 \widetilde{S}+1)^{-1}$ for $\widetilde{S}=S$ $-1 / 2$, while it does not split for $\widetilde{S}=S+1 / 2$.

Examples of the normalized current $F_{I}$ are plotted as a function of the emitter chemical potential $\mu_{e}$ for several values of the spin splitting for $S=0, \widetilde{S}=1 / 2$ in Fig. 1 and for $S=1 / 2, \widetilde{S}=1$ in Fig. 2 . For both cases, the results are given for the positive bias with $\mu_{c}$ fixed well below the addition energies and for the negative bias with $\mu_{c}$ well above them. At large spin splittings, there appears one or two steps depending on the bias polarity as described above. The step width here is determined by the thermal energy $k_{B} T$. The result demonstrates that the total spin can be determined from the universal dependence $F_{I}$, when it is observed, us- 
ing the above formulas. The ratio of the spin-split step heights can be determined even from data of low magnetic fields, since curves for different magnetic fields cross nearly at the same point.

In conclusion, we have shown that the relative step heights of the Coulomb staircase provides a simple method to determine the total spin of the many-body ground state in a quantum dot. The first example is to use the difference in height for different bias polarities and the second is to use the spin-split steps due to the in-plane magnetic field. The knowlegde of the total spin is essential to confirm the spin blockade experimentally. To identify the spin blockade at the transition between $N_{0}$ and $N_{0}+1$ electrons in the dot, we need to know the spins of the corresponding ground states. We can determine the spin of the $N_{0}$-electron ground state from the current step due to the transition between $N_{0}-1$ and $N_{0}$ and that of the $N_{0}+1$-electron ground state from the step between $N_{0}+1$ and $N_{0}+2$.
The in-plane magnetic field producing the spin splitting with little change in orbits is a useful tool in studying manybody effects in transport through a quantum dot and has already been employed in several experiments. ${ }^{17,8}$ Influences of the spin splitting on tunnelings have been theoretically studied for a single orbit ${ }^{18}$ and for more than one orbit, ${ }^{22}$ and shown to be useful in clarifying the Coulomb correlation between tunnelings of electrons with opposite spins, ${ }^{18,22}$ the dephasing due to spin flips, ${ }^{22}$ and the spin blockade as described in this paper.

The author would like to thank N. Tokuda and Y. Asano for valuable discussions. This work was supported in part by a Grant-in-Aid for Scientific Research on Priority Area "Single Electron Devices and Their High Density Integration" from the Ministry of Education, Science, Sports and Culture, Japan.
${ }^{1}$ M.A. Kastner, Phys. Today 46 (1), 124 (1993). Rev. Mod. Phys. 64, 849 (1992).

${ }^{2}$ L. P. Kouwenhoven, C.M. Marcus, P.L. McEuen, S. Tarucha, R.M. Westervelt, and N.S. Wingreen, in Mesoscopic Electron Transport, edited by L.L. Sohn, L.P. Kouwenhoven, and G. Schön (Kluwer, Dordrecht, The Netherlands, 1997).

${ }^{3}$ B. Su, V.J. Goldman, and J.E. Cunningham, Phys. Rev. B 46, 7644 (1992).

${ }^{4}$ R.C. Ashoori, H.L. Stormer, J.S. Weiner, L.N. Pfeiffer, K.W. Baldwin, and K.W. West, Phys. Rev. Lett. 71, 613 (1993).

${ }^{5}$ S. Tarucha, D.G. Austing, T. Honda, R.J. van der Hage, and L.P. Kouwenhoven, Phys. Rev. Lett. 77, 3613 (1996).

${ }^{6}$ D.R. Stewart, D. Sprinzak, C.M. Marcus, C.I. Duruöz, J.S. Harris, Jr., Science 278, 1784 (1997).

${ }^{7}$ L.P. Kouwenhoven, T.H. Oosterkamp, M.W.S. Danoesastro, M. Eto, D.G. Austing, T. Honda, and S. Tarucha, Science 278, 1788 (1997).

${ }^{8}$ D. Goldhaber-Gordon, H. Shtrikman, D. Mahalu, D. AbuschMagder, U. Meirav, and M.A. Kastner, Nature (London) 391, 156 (1998).

${ }^{9}$ S.M. Cronenwett, T.H. Oosterkamp, and L.P. Kouwenhoven, Science 281, 540 (1998).

${ }^{10}$ H. Akera, Phys. Rev. B 47, 6835 (1993); Jpn. J. Appl. Phys., Part 1 36, 3974 (1997); Solid-State Electron. 42, 1379 (1998); Jpn. J. Appl. Phys., Part 1 38, 384 (1999).

${ }^{11}$ D. Weinmann, W. Häusler, W. Pfaff, B. Kramer, and U. Weiss, Europhys. Lett. 26, 467 (1994).

${ }^{12}$ D. Weinmann, W. Häusler, and B. Kramer, Phys. Rev. Lett. 74, 984 (1995).
${ }^{13}$ Y. Tanaka and H. Akera, Phys. Rev. B 53, 3901 (1996).

${ }^{14}$ M. Eto, J. Phys. Soc. Jpn. 66, 2244 (1997); Solid-State Electron. 42, 1373 (1998).

${ }^{15}$ H. Imamura, H. Aoki, and P.A. Maksym, Phys. Rev. B 57, R4257 (1998).

${ }^{16}$ Y. Asano, Phys. Rev. B 58, 1414 (1998); J. Phys. Soc. Jpn. 67, 4014 (1998).

${ }^{17}$ J. Weis, R.J. Haug, K.v. Klitzing, and K. Ploog, Phys. Rev. Lett. 71, 4019 (1993).

${ }^{18}$ L.I. Glazman and K.A. Matveev, Pis'ma Zh. Éksp. Teor. Fiz. 48, 403 (1988) [JETP Lett. 48, 445 (1988)].

${ }^{19}$ An observed Coulomb staircase showing such a dependence on the bias polarity and its analysis based on spin degeneracy have been reported (Ref. 20).

${ }^{20}$ D.H. Cobden, M. Bockrath, P.L. McEuen, A.G. Rinzler, and R.E. Smalley, Phys. Rev. Lett. 81, 681 (1998).

${ }^{21}$ Similar universal dependences have been found for the conductance and the transmission coefficient through a quantum dot (Ref. 22).

${ }^{22}$ H. Akera, Phys. Rev. B 59, 9802 (1999).

${ }^{23}$ C.W.J Beenakker, Phys. Rev. B 44, 1646 (1991).

${ }^{24}$ D.V. Averin, A.N. Korotkov, and K.K. Likharev, Phys. Rev. B 44, 6199 (1991).

${ }^{25}$ Y. Kuramoto, Z. Phys. B 53, 37 (1983).

${ }^{26}$ N.E. Bickers, Rev. Mod. Phys. 59, 845 (1987).

${ }^{27}$ D.C. Ralph, C.T. Black, and M. Tinkham, Phys. Rev. Lett. 74, 3241 (1995); 78, 4087 (1997).

${ }^{28} \mathrm{~A}$ method to determine the total spin by using the relative strengths of spin-split peaks in $d I / d V$ plot has been mentioned briefly (Ref. 20). 